\title{
Atomistic view on thin film nucleation and growth by using highly ionized and pulsed vapour fluxes
}

\author{
Kostas Sarakinos, Daniel Magnfält, Viktor Elofsson and B. Lu
}

\section{Linköping University Post Print}

\section{Tweet}

N.B.: When citing this work, cite the original article.

Original Publication:

Kostas Sarakinos, Daniel Magnfält, Viktor Elofsson and B. Lu, Atomistic view on thin film nucleation and growth by using highly ionized and pulsed vapour fluxes, 2014, Surface \& Coatings Technology, (257), 326-332.

http://dx.doi.org/10.1016/j.surfcoat.2014.04.015

Copyright: Elsevier

http://www.elsevier.com/

Postprint available at: Linköping University Electronic Press

http://urn.kb.se/resolve?urn=urn:nbn:se:liu:diva-112642 
Atomistic view on thin film nucleation and growth by using highly ionized and pulsed vapour fluxes

K. Sarakinos, D. Magnfält, V. Elofsson, and B. Lü

Plasma and Coatings Physics Division, Department of Physics, Chemistry and Biology (IFM), Linköping University, SE-58183 Linköping, Sweden

\begin{abstract}
We present a brief review on the use of ionized and pulsed vapour fluxes, primarily generated by high power impulse magnetron sputtering (HiPIMS) discharges, as tools to gain atomistic understanding on film nucleation and growth. Two case studies are considered; the first case study concerns stress generation in polycrystalline films. It is highlighted that by using vapour fluxes of well-controlled ion content and ion energy and by studying the film microstructure and intrinsic stresses one can obtain experimental evidence for stress generation by insertion of film forming species in the grain boundaries. In the second case study it is discussed how the use of pulsed vapour fluxes with well controlled time domain can facilitate understanding of growth dynamics and microstructural evolution in thin films grown in three-dimensional (i.e., Volmer-Weber) fashion. Broader implications of the described research strategies for the surface science and surface engineering communities are highlighted and discussed.
\end{abstract}




\section{Introduction}

The majority of thin films is nowadays synthesized by vapour condensation on solid surfaces [1,2]. During the synthesis process, relatively high arrival rates of film forming species (typically of the order of $10^{13} \mathrm{~cm}^{-2} \mathrm{~s}^{-1}$ or equivalently $10^{-2} \mathrm{MLs}^{-1}$ and above) and deposition at homologous temperatures $\left(T_{s} / T_{m}\right)$ below 0.3 result in limited atomic assembly kinetics [3]. The latter in combination with extremely high cooling rates $\left(\sim 10^{13} \mathrm{Ks}^{-1}\right)$ encountered during the condensation of the vapour on the substrate surface [4] lead to growth far from thermodynamic equilibrium which, in turn, makes it possible to form metastable phases and microstructures with unique attributes $[3,5,6]$.

A significant part of thin film synthesis processes and the related applications involve polycrystalline films, i.e. films that consist of crystallites (grains) with different crystallographic orientations. The growth of polycrystalline films starts with the formation of spatially separated single-crystalline islands (nucleation) which grow in size (island growth) and impinge on each other forming new, larger islands (coalescence). This process also leads to reduction of the island density on the substrate surface. The coalescence process continues until the boundaries between single-crystalline islands (i.e. grains) become immobile and coalescence cannot be completed (formation of polycrystalline islands) which lead to the formation of a continuous film [3]. The microstructural characteristics and evolution during these growth stages are dictated by the effect of the synthesis conditions (in terms of thermodynamics and kinetics) on the three fundamental structure forming phenomena, namely nucleation, crystal growth and grain growth [7]. Understanding how thermodynamics and, primarily, kinetics affect these phenomena on a 
fundamental level is of vital importance for design and synthesis of thin film materials in a tailor-made fashion. The surface science and surface engineering communities seek to achieve this understanding by combining in situ tools that allow for real-time monitoring of deposition processes and film growth evolution, ex situ characterization tools that provide microstructural information at the nanoscale and growth simulations $[2,3,8]$. The characteristics of the vapour flux used for thin film synthesis are also an important factor in the quest for an atomistic understanding of thin film growth. For instance, vapour fluxes that partially or fully consist of ionized species can be used to ion-irradiate the growing film in a controlled manner (by coupling those fluxes with electric and/or magnetic fields to tune the energies and trajectories of the charged particles) and, thus, trigger, enhance and reveal surface and subsurface processes that control kinetically limited film microstructural evolution $[3,9]$. Another example is pulsed vapour fluxes with well-controlled time domain that can be employed to understand the dynamics of the film growth [10-14]. The present contribution is a brief review of recent studies where pulsed and ionized vapour fluxes, primarily generated by a plasma-based technique termed high power impulse magnetron sputtering (HiPIMS), are used to contribute to the fundamental understanding of thin film growth. The paper focuses on two case studies; (i) surface and sub-surface processes that lead to generation of intrinsic stresses in polycrystalline films (section 3) and (ii) dynamics of microstructural evolution during three-dimensional thin film growth (section 4). Prior to presenting the case studies mentioned above, a short overview of different approaches to generate pulsed and ionized vapour fluxes is presented (section 2). It should be brought to reader's attention that parts of sections 3, 4 are built upon results presented in two recently published works by Magnfält et 
al. $[15,16]$ by putting these results in to a broader context as compared to the original reports.

\section{Strategies for generation of highly ionized and pulsed vapour fluxes}

Generation of highly ionized fluxes can be achieved by glow discharges (plasmas) that are characterized by high electron density and temperature [17]. These discharge conditions are accessible by a variety of plasma-based physical vapour deposition techniques, such as pulsed laser deposition (PLD) [18], filtered cathodic arc (FCA) [19], inductively coupled plasma assisted discharged [20,21] or microwave assisted magnetron sputtering discharges [22-25]. Magnetic coils in combination with unbalanced magnetrons can also be used to tune the discharge gas ion flux for ion assisted film growth [26]. Another approach that facilitates ion assisted growth is the simultaneous use of neutral vapour fluxes generated by physical means and charged fluxes generated by ion sources [27]. Well controlled energetic bombardment using ionized fluxes has been utilized in an attempt to understand the fundamental atomistic processes that determine, for instance, nucleation in epitaxial and polycrystalline films [28-35], texture and microstructural evolution in transition metal nitrides [9,36,37], phase formation in alumina [38-40], structure formation (i.e., bonding characteristics) in amorphous carbon [41], and growth evolution of metallic films [42].

Vapour fluxes with a periodic temporal profile can be in practice created using a continuous plasma source (e.g., a thermal evaporator) equipped with a rotating mechanical shutter [43]. The time-dependent character of those fluxes has, in conjunction with growth simulations, been used to understand and manipulate the 
dynamics of nucleation processes during epitaxy experiments and improve properties of functional films [10, 43-45]. Alternatively, pulsed plasma sources, such as PLD, can also generate pulsed vapour fluxes [11,46]. Again, the pulsed character of deposition fluxes generated by PLD discharges has been used to control films nucleation [47] and growth [48] as well as to understand the dynamics of island coalescence and its effect of the film microstructural evolution during Volmer-Weber film growth [11].

In 1999 Kouznetsov et al. [49], inspired by previous work in former USSR [50-52] developed a sputtering based ionized physical vapour deposition technique, termed high power impulse magnetron sputtering (HiPIMS). In HiPIMS power is applied to a sputtering cathode in short (typically less than $150 \mu \mathrm{s}$ ), unipolar pulses of low duty cycle $(<10 \%)$ and frequency (up to few $\mathrm{kHz}$ ). This mode of operation results in power density values during the pulse on-time (termed peak power density) of the order of several $\mathrm{kWcm}^{-2}$, which in turn yield plasma densities of the order of $10^{19}$ particles $/ \mathrm{m}^{3}$ and ionized fractions of up to $100 \%$ of the sputtered material [53-58]. In addition, by navigating within the HiPIMS parameter space (e.g., by changing the peak target power density) one is able to tune the ionization degree and the energy of the ionized film forming species [53-58]. In the course of the past 15 years, the highly ionized fluxes generated by HiPIMS have been used to grow dense and smooth films, engineer their microstructure and the film-substrate interface and control their phase composition and other functional properties $[54,55,57]$. Less work has been done on utilizing the highly ionized fluxes for understanding film nucleation and growth on a fundamental level with the exception of sporadic attempts to study structure formation 
in transition metal oxides [59], growth evolution in transition metal nitrides [60-63] and microstructural evolution during epitaxial film growth [64].

At the same time, HiPIMS is a pulsed plasma-based process in which pulse width, frequency and amplitude can be controlled independently, i.e., exhibits the potential to generate pulsed fluxes with very well controlled time-domain $[65,66]$. This paper reviews recent attempts to use pulsed and highly ionized vapour fluxes, primarily generated by HiPIMS discharges, as tools to contribute to the understanding of intrinsic stress generation in polycrystalline films (section 3) and the dynamics of microstructural evolution during three-dimensional (Volmer-Weber) film growth (section 4).

\section{Surface and sub-surface processes leading to stress generation in polycrystalline films deposited from the vapour phase}

Stresses are a common feature in thin films and they are paramount to film functionality, since they largely determine film adhesion on underlying substrates [6769]. Intrinsic stresses are generated when strain is imposed upon a thin film that is constrained on a substrate [1]. Sources of strain (and thus of intrinsic stresses) are volume changes induced by crystallization [70] or phase transformations [71, 72]. For example, in $\mathrm{Al}_{2} \mathrm{O}_{3}$ films, transformation of the metastable $\gamma-\mathrm{Al}_{2} \mathrm{O}_{3}$ phase into the thermodynamically stable $\alpha-\mathrm{Al}_{2} \mathrm{O}_{3}$ phase upon heating results in a reduction of the molar volume by $\sim 8 \%[71,72]$. The latter, in turn, gives rise to tensile stress generation and crack formation [71, 72]. Other sources of stress/strain include thermal stresses generated by differences in the thermal expansion coefficient 
between film and substrate $[67,69]$ and coherency stresses generated at the filmsubstrate interface during local or large-scale heteroepitaxial growth [1].

Polycrystalline films grown from the vapour phase are subjected to the stress mechanisms described above but often also exhibit growth-induced stresses that are generated in the grains and in the grain boundaries $[67,69]$. Compressive stresses are typically observed before film coalescence and it is widely agreed upon that they originate from Laplace pressure leading to a smaller than bulk lattice spacing frozen in during island growth $[73,74]$. During the coalescence stage neighbouring grains deform elastically to form grain boundaries as it is favourable to trade surface energy for strain and grain boundary energy [75-84]. This process causes tensile stresses (Fig.1) [75-84]. This stress state prevails after the completion of the island coalescence in the continuous growth stage when films are deposited at relatively low homologous temperatures, $T_{s} / T_{m}$ (typically well below 0.2 ), that favour the formation of columnar structures with underdense grain boundaries, i.e., porous grain boundaries with mass density significantly smaller to that of the grains (see right panel in Fig.1) [3]. Concurrent bombardment by energetic species (e.g. ions) causes creation of point defects (e.g., interstitials and vacancies) that lead to out-of-plane film lattice expansion and compressive stress generation [85-89]. Compressive stresses generated in the grains and tensile stresses due to the grain boundary shrinkage are independent and additive [85]. When deposition is performed at relatively high $T_{s} / T_{m}$ (typically above 0.2 ) adatoms have sufficient mobility for formation of dense films to occur [3]. This is accompanied by generation of a compressive growth stress after formation of a continuous film which relaxes on deposition interruptions $[67,80]$ as seen in the left panel in Fig.1. Using in situ 
monitoring of intrinsic stress evolution during and after deposition, growth simulations and analytical models, a number of research groups [77, 78, 90-95] have attributed compressive stress generation to diffusion of adatoms into grain boundaries. The driving force for this diffusion has been suggested to be a chemical potential difference between the surface of the growing film (which is supersaturated with adatoms) and the grain boundaries (which are depleted from atoms) [77, 78, 95]. The stress relaxation was then argued to be the effect of out-diffusion of atoms from grain boundaries due to a reversal of the chemical potential difference when deposition is interrupted $[77,78,95]$. Experimental studies have reported that the stress relaxation upon interruption scales with the inverse grain size as predicted by the out-diffusion model [96] but that the stress relaxation does not have the temperature dependence predicted by a diffusion based relaxation process [97, 98]. Others have suggested $[81,99]$ that grain rotation coupled with grain boundary diffusion as a cause of the stress relaxation. Alternative explanations for the compressive stress build up include recovery of the Laplace pressure induced stress after coalescence completion [100] and trapping of atoms between surface ledges [73], while stress relaxation has been also attributed to surface flattening and grain growth [100]. It has also been suggested that compressive stress build up and generation is due to changes in surface stresses when adatom concentration is varied during growth [101-103]. However, it has been argued that stresses induced by these mechanisms are too small to explain changes in stress magnitude observed experimentally [104].

One way to seek evidence for insertion of film forming species into grain boundaries is by studying the film microstructure and attempt to correlate it with the intrinsic stress evolution post-deposition. However, the post-deposition stress relaxation at 
high mobility (i.e., high $T_{s} / T_{m}$ ) (see left panel in Fig.1) makes a correlation between stress and film microstructure a difficult task. Elimination or minimization of the stress relaxation requires relatively low $T_{s} / T_{m}$ values which result in underdense microstructures with open grain boundaries that yield tensile stress. The only studies available in the literature in which surface diffusion is active leading to compressive stress generation and no relaxation on deposition interruption is observed concern epitaxial growth of AIN epitaxial columns on $\mathrm{Si}$ (111) [105].

A way to circumnavigate the limitations described above and to establish the relationships between film stress state and microstructure is to freeze the state of the film at the completion of the deposition. Magnfält et al. [15] argued that this can be achieved by employing growth conditions that facilitate sufficient atomic mobility on the surface and in near-surface layers during deposition but result in negligible postdeposition surface and bulk mobility. Bulk and surface mobility can be suppressed by depositing films at relatively low $T_{s} / T_{m}$ values. Concurrent bombardment of the growing surface by large fluxes of hyperthermal species that possess moderate energies (several 10s up to $\sim 100 \mathrm{eV}$ ), can affect the first atomic layers of the film and trigger diffusion processes [3]. Using highly ionized energetic vapour fluxes generated by a HiPIMS discharge Magnfält et al. [15] grew Mo films at ambient temperature ( $\left.T_{s} / T_{m} \sim 0.13\right)$. The energy and the flux (amount) of ionized species were controlled by changing the peak power density $\left(P_{T p d}\right)$ on the Mo sputtering cathode. The effect of the energetic bombardment conditions on the intrinsic stress $(\sigma)$ and the stress-free lattice parameter $\left(a_{0}\right)$ of the films measured using the $\sin ^{2} \psi$ method adapted for textured layers is shown in Fig.2. There it is seen that $\sigma$ (left axis in Fig.2) takes values from +0.2 to $-3 \mathrm{GPa}$ with increasing $P_{T p d}$ (i.e., larger energies and fluxes 
of bombarding species). This behaviour is commonly observed in polycrystalline films grown under bombardment by hyperthermal species. These species cause generation of point defects which, in turn, leads to a hydrostatic strain field that manifests itself as a larger than bulk stress free lattice parameter, ao $[85,86,89$, 106]. However, despite the differences in the stress sign and magnitude, ao is nearly constant for the various $P_{T p d}$ values used for the film growth and only marginally larger $(0.12 \%)$ than the stress-free lattice parameter of bulk Mo [107] (horizontal dashed line in Fig.2). The findings in Fig.2 are clear evidence that the differences in stress sign and magnitude between the films cannot be attributed to changes in defect concentration in the grains (that cause hydrostatic stress). Instead the film stress is mainly biaxial. By correlating this with the evolution of the film mass density and grain size Magnfält et al. [15] concluded that stresses are generated by grain boundary densification. The latter is, in turn, caused by the energetic ionized species that trigger diffusional incorporation of film forming species into grain boundaries [15], analogous to what has been suggested by Chason et al. $[78,79]$ for films deposited in the absence of energetic depositing species. Further evidence for correlation between grain boundary densification and compressive stress generation at relatively low $T_{s} / T_{m}$ values can be found in the data reported by Koutsokeras and Abadias [94] who combined in situ and ex situ techniques to study the contribution of grains and grain boundaries to stress generation in $\mathrm{ZrN}$ films grown by magnetron sputtering. By varying the working pressure during deposition the energy transferred from plasma species to the growing film was altered. Grains and grain boundaries were found to be in compressive stress state at conditions that corresponded to intense energetic bombardment (i.e., relatively low working pressure) with grain boundaries exhibiting density close to that of the grains. On the contrary, conditions that corresponded to 
less intense energetic bombardment (i.e., relatively high working pressure) grain boundaries were found to be underdense and in tensile stress state. Analogous to the additivity of compressive stresses generated by energetic bombardment induced point defects in the grain bulk and tensile stresses generated in the grain boundaries $[85,86]$, it can be expected that compressive stresses generated in grain boundaries are additive with compressive stresses generated by point defects. However, this is still lacking experimental verification.

\section{Dynamics of microstructural evolution during Volmer-Weber thin film growth} Polycrystalline films with surface energy larger than that of the underlying substrate tend to grow in the so-called Volmer-Weber mode (or 3-dimensional island mode) [1, 2] that makes the distinction between the different growth stages described in section 1 particularly pronounced. The island density achieved during the nucleation stage and the dynamics of island growth and coalescence are decisive for the microstructural evolution during growth and hence for film properties, such as roughness, grain size, film thickness at percolation (i.e. when a metallic film conducts over macroscopic distances) and thickness at which a continuous film is formed [1]. The steady-state island density, $N$, is determined by the kinetic conditions during growth and in the case of deposition using a temporally continuous flux can be expressed by the scaling relation [8]

$$
N \propto\left(\frac{F_{a v}}{D}\right)^{\chi}
$$

In Eq. (1) $\chi$ is a scaling exponent which in the case of 3-dimensional nucleation is calculated as [108]:

$$
\chi=\frac{i^{*}}{i^{*}+2.5}(2)
$$


where $i^{\star}$ is the critical nuclei size. For low temperature deposition $i^{\star}=1$, i.e. dimers are the smallest stable nuclei and Eq. (2) yields a scaling exponent of $\sim 0.286$. The other parameters in Eq. (1) are the time-average deposition flux $\left(F_{a v}\right)$ and the adatom diffusivity $(D)$. The latter depends exponentially on the growth temperature $T$ and the energy barrier for adatom surface diffusion, $E_{D}$, through the expression [8]

$$
D \propto \exp \left(-\frac{E_{D}}{k_{B} T}\right)(3)
$$

where $k_{B}$ is the Boltzmann constant. From Eqs. (1) and (3) it becomes evident that relatively small variations of $T$ induce relatively large changes in $N$. This is in contrast to $F_{a v}$, which needs to be varied over many orders of magnitude to affect $N$ to the same degree, as depicted in Fig.3.

An additional kinetic handle to control the nucleation process can become available by modulating a given time-averaged $\left(F_{a v}\right)$ deposition flux in pulses of well-defined width (ton), frequency $(f)$ and amplitude $\left(F_{p}\right)$. The effect of flux modulation on nucleation depends on the relation between the adatom lifetime $\left(\tau_{m}\right)-$ i.e., the mean time that an adatom needs to get incorporated into a stable island - and the time scale of the deposition flux [10]. Jensen and Niemeyer have defined three time scale/adatom diffusivity regimes [10] (Fig.4), namely:

(i) Slow diffusivity regime $\left(\tau_{m}>>1 / f\right)$. In this regime the adatom density does not vanish between the vapour pulses, in other words the substrate encounters a continuous deposition flux and the island density scales according to Eq. (1).

(ii) Fast diffusivity regime $\left(\tau_{m}<<t_{o n}\right)$. In this regime adatom condensation, adatom diffusion and island formation happen within a single vapour pulse which leads to an effectively "continuous" flux with vapour arrival rate equal to $F_{p}$, i.e. in this case the island density scales as 


$$
N \propto\left(\frac{F_{p}}{D}\right)^{0.286}
$$

(iii) Intermediate diffusivity regime $\left(t_{o n}<\tau_{m}<1 / f\right)$. In this regime the adatom diffusion time is longer than the vapour pulse, i.e., during the pulse on-time there is not enough time for diffusion to become the rate-limiting step for the nucleation density [8] which in this case scales as

$$
N \propto\left(F_{p} t_{o n}\right)^{1 / 2}
$$

From the discussion presented above it becomes evident that nucleation can be tailored in pulsed deposition processes by appropriate selection of flux time scales (ton and $f$ ) and instantaneous deposition rate $\left(F_{p}\right)$.

The nucleation is stage is followed by the island growth stage which leads to island impingement and coalescence. The island impingement rate is determined by the rate at which the islands grow which is, in turn, a function of the time-averaged vapour flux. The rate of coalescence is a function of the island size, $R$, according to the expression [109]

$$
\frac{1}{\tau_{\text {coal }}}=\frac{B}{R^{4}}(6)
$$

where $B$ is a temperature and material dependent constant. Equation (6) implies that the coalescence rate decreases with the size of the island, while impingement events become more frequent the larger the islands become. As film growth proceeds there is a point at which the two rates (impingement and coalescence) become equal, i.e., an island impinges onto a coalescing cluster before coalescence is completed [110, 111]. This leads to the formation of elongated features separated by voids. The film thickness at which this happens is termed the elongation transition thickness ( $\left.d_{\text {elong }}\right)$ $[110,111]$ and it is, by convention, used to signify the onset of the transition towards 
a continuous film $[110,111]$. It is in general difficult to determine $d_{\text {elong }}$ experimentally because it routinely lies in ranges that are not accessible by conventional analytical techniques. Instead $d_{\text {elong }}$ can be calculated from analytical models and growth simulations. In case of film growth using continuous vapour fluxes both analytical models and kinetic Monte-Carlo (KMC) simulations have shown that $d_{e l o n g}$ scales with the average vapour flux $F_{a v}$ and the coalescence parameter $B$ according to the relation

$$
d_{\text {elong }} \propto\left(\frac{F_{a v}}{B}\right)^{-1 / 3}
$$

When instead pulsed vapour fluxes are used Warrender and Aziz [11] have shown that scaling exponents (cf. Eq. (7)) between 0 and -0.44 can be obtained depending on the pulsing frequency and the surface diffusivity conditions using KMC simulations. Warrender and Aziz [11] argued that the scaling regimes are result of the interplay between the temporal profile of the deposition flux (i.e., pulsing frequency), the adatom lifetime and the coalescence time and their effect on nucleation and coalescence characteristics. They also found a good consistency between the $d_{\text {elong }}$ data obtained from KMC and percolation transition thickness determined using in situ resistivity measurements during the growth of $\mathrm{Ag}$ films on $\mathrm{SiO}_{2}$ substrates by pulsed laser deposition (PLD) [11]. PLD processes generate a highly ionized deposition flux with ion energies up to $300 \mathrm{eV}$ [18]. These energetic ions affect both surface and subsurface layers and thus film nucleation and coalescence $[3,15,28,29,112]$. The broad ion energy distribution functions (IEDFs) make identification and understanding of the atomistic mechanisms that along with the modulated vapour flux determine the film microstructural evolution a non-trivial task. To improve this understanding, sources that generate ionized vapour fluxes with relatively narrow IEDFs as well as sources that generate pulsed vapour fluxes with well-controlled time domain are of 
benefit. HiPIMS exhibits the potential to meet these requirements since it generates ions with distribution functions in the range of $10 \mathrm{~s}$ of $\mathrm{eV}$ if appropriate pulsing parameters are used [53-58]. Using this type of fluxes Magnus et al. [60] have found that nucleation and coalescence processes of $\mathrm{TiN}$ films grown on $\mathrm{SiO}_{2}$ can be affected by a more intense energetic bombardment leading lower film resistivity as compared to film grown by dc magnetron sputtering. Lattemann et al. [61] argued that it is not only the bombardment by the energetic ions that affects the nucleation process but also the high instantaneous rate during the vapour pulse which lead to an increased supersaturation and high nucleation density. The latter in combination with long times between the pulses allows for adatoms to diffuse towards low energy sites giving rise to smooth and dense TiN (111) oriented films [61]. Moreover, Mitschker et al. [66] have shown that HiPIMS can generate temporally modulated fluxes while Magnfält et al. [16] have shown that the time-domain of Ag fluxes can be controlled by the frequency of the power applied to the sputtering cathode. It was also shown that by keeping the energy during the power pulse constant the temporal profile of the flux can be varied without changing the $\mathrm{Ag}^{+}$IEDFs, i.e. the effect of flux modulation can be decoupled by that of energetic bombardment and instantaneous deposition rate [16]. Using pulsed vapour fluxes generated by this methodology they studied the effect of the flux time domain on thickness at which a continuous Ag film is formed on $\mathrm{SiO}_{2}$ substrates by means of in situ spectroscopic ellipsometry and in situ stress measurements [16]. The results (presented in Fig.5) are qualitatively consistent with those reported by Warrender et al. [11]. Moreover, comparison with films grown using a continuous flux (also presented in Fig.5) within the same average growth rate range showed that they (i.e., films deposited using a continuous flux) became continuous at larger thicknesses. Magnfält et al. [16] also estimated the $\mathrm{Ag}$ 
adatom lifetime and coalescence time and found both to lie within the time scale (i.e. frequency) of the deposition flux which led them to the conclusion that both processes (i.e. nucleation and coalescence) are likely to be affected by the deposition flux modulation.

\section{Summary and implications for surface science and engineering}

In the present brief review we described how ionized and pulsed vapour fluxes can be used to gain atomistic understanding on the fundamental processes that control film nucleation and growth. Well controlled (with respect to their energy and ionization faction) ionized vapour fluxes have been found to cause film (i.e., grain boundary) densification and generation of biaxial compressive stresses in Mo films. Moreover, the stress state is maintained after deposition interruption, in contrast to what is observed in high-mobility metals. The correlation between film densification and compressive stresses provides clear evidence that compressive stresses in Mo films can be generated by diffusional insertion of film forming species into grain boundaries. These data can be of relevance for explaining stress generation in lowmobility refractory films. They also imply that densification is achieved at the cost of large compressive stress generation, a notion that can be used to tailor and/or mitigate film stresses. The suggested mechanisms may also be of relevance for shedding light on the densification of films when using highly ionized fluxes, as commonly observed in thin films deposited using HiPIMS [53-55]. This will allow for understanding why densification happens not only in terms of plasma conditions [113] but also in terms of intrinsic material properties. Thus, appropriate synthesis strategies for film densification can be designed in a knowledge-based manner. 
It was also shown that pulsed fluxes with well-controlled time domain can be used to understand and control growth dynamics of $\mathrm{Ag}$ films deposited on $\mathrm{SiO}_{2}$ which is a well-studied archetype system for Volmer-Weber growth. The well-controlled time domain can also be used as input in and to benchmark growth simulations that in turn can provide information about the significance of each growth stage on film microstructural evolution. Using this notion one would be able to design growth strategies to optimize film properties and performance in technological applications, e.g., optimizing optical and electrical properties of Ag films in low-emissivity windows. The pulsing frequency dependence on the microstructural evolution can also be used as a tool to determine effective diffusion and coalescence times. By measuring and observing the evolution of the island density at different growth stages, conditions at which characteristic times for diffusion and coalescence become comparable to the time-scale of the modulation of the vapour flux can be accurately identified. This, in turn, can be used to determine surface diffusion and coalescence time constants at various growth conditions.

\section{Acknowledgements}

The authors should like to acknowledge financial support from Linköping University through the "LiU Research Fellows" program, the Swedish Research Council through the contract VR 621-2001-5312 and Ångpanneföreningens Forskningsstiftelse through the project "Towards Next Generation Energy Saving Windows". 


\section{References}

[1] M. Ohring, Materials Science of Thin Films, Academic Press, San Diego (1991).

[2] P. M. Martin (Ed.), Handbook of Deposition Technologies for Films and Coatings, Elsevier, Amsterdam (2005).

[3] I. Petrov, P.B. Barna, L. Hultman, and J.E. Greene, J. Vac. Sci. Technol. A 21 (2003) S117.

[4] T.W. Barbee, W.H. Holmes, D. L. Keith, M.K. Pyzyna, and G. Ilcona, Thin Solid Films 45 (1977) 591.

[5] W.-D. Münz, J. Vac. Sci. Technol. A 4 (1986) 2717.

[6] F. Rovere, D. Music, S. Ershov, M. to Baben, H.-G. Fuss, P.H. Mayrhofer, and J.M. Schneider, J. Phys. D: Appl. Phys. 43 (2010) 035302.

[7] P.B. Barna and M. Adamik, Thin Solid Films 317 (1998) 27.

[8] T. Michely and J. Krug, Island Mounds and Atoms, Springer, Berlin (2005).

[9] P. Patsalas, C. Gravalidis, and S. Logothetidis, J. Appl. Phys. 96 (2004) 6234.

[10] P. Jensen and B. Niemeyer, Surf. Sci. 384 (1997) L823.

[11] J. M. Warrender and M. Aziz, Phys. Rev. B 76 (2007) 045414.

[12] B. Hinnemann, H. Hinrichsen, and D. Wolf, Phys. Rev. Lett. 87 (2001) 22.

[13] N. Combe and P. Jensen, Phys. Rev. B 57 (1998) 553.

[14] L. Guan, D. Zhang, X. Li, and Z. Li, Nucl. Instruments Methods Phys. Res. Sect.

B Beam Interact. with Mater. Atoms, 266 (2008) 57-62.

[15] D. Magnfällt, G. Abadias, and K. Sarakinos, Appl. Phys. Lett. 103 (2013) 051910.

[16] D. Magnfält, V. Elofsson, G. Abadias, U. Helmersson, and K. Sarakinos, J. Phys. D: Appl. Phys. 46 (2013) 215303. 
[17] J.A. Hopwood (Ed.), Ionized Physical Vapor Deposition, Academic Press, San Diego (2000).

[18] J. M. Warrender and M. Aziz, Phys. Rev B. 75 (2007) 085433.

[19] A. Anders, Cathodic Arcs: From Fractal Spots to Energetic Condensation, Springer, Berlin (2009).

[20] S.M. Rossnagel, J. Hopwood, J. Vac. Sci. Technol., B 12 (1994) 449.

[21] S.M. Rossnagel, J. Hopwood, Appl. Phys. Lett. 63 (1993) 3285.

[22] J. Musil, S. Kadlec, W.-D. Münz, J. Vac. Sci. Technol., A, Vac. Surf. Films 9 (1991) 1171.

[23] J. Xu, X. Deng, J. Zhang, W. Lu, T. Ma, Thin Solid Films 390 (2001) 107.

[24] A. Yonesu, T. Kato, H. Takemoto, N. Nishimura, Y. Yamashiro, Jpn. J. Appl. Phys. 38 (1999) 4326.

[25] C. Boisse-Laporte, O. Leroy, L. de Poucques, B. Agius, J. Bretagne, M.C. Hugon, L. Teulé-Gay, M. Touzeau, Surf. Coat. Technol. 179 (2004) 176.

[26] I. Petrov, F. Adibi, J. E. Greene, W. D. Sproul, and W.-D. Munz, J. Vac.Sci. Technol. A 10 (1992) 3283.

[27] B. Rauschenbach and J. W. Gerlach, Crystal Research and Technology, 35 (2000) 675 .

[28] M. Kalff, M. Breeman, M. Morgenstern, T. Michely, and G. Comsa, Appl. Phys. Lett. 70 (1997) 182.

[29] S. Esch, M. Bott, T. Michely, and G. Comsa, Appl. Phys. Lett. 67 (1995) 3209.

[30] B. Degroote, A. Vantomme, H. Pattyn, and K. Vanormelingen, Phys. Rev. B 65 (2002) 195401.

[31] H.-A. Durand, K. Sekine, K. Etoh, K. Ito, and I. Kataoka, Thin Solid Films 336 (1998) 42. 
[32] H.-A. Durand, K. Sekine, K. Etoh, K. Ito, and I. Kataoka, Surf. Coat. Technol. 125 (2000) 111.

[33] A. Romanyuk, R. Steiner,V. Thommen, P. Oelhafen, and D. Mathys, J. Appl. Phys. 100 (2006) 074904.

[34] R. Ditchfield and E.G. Seebauer, Phys. Rev. Lett. 82 (1999) 1185.

[35] R. Ditchfield and E.G. Seebauer, Phys. Rev. B 63 (2001) 125317.

[36] C.S. Shin, Y.W. Kim, N. Hellgren, D. Gall, I. Petrov, and J.E. Greene, J. Vac. Sci. Technol. A 20 (2002) 2007.

[37] D. Gall, C.S. Shin, T. Spila, M. Odén, M.J.H. Senna, J. E. Greene, and I. Petrov, J. Appl. Phys. 91 (2002) 3589.

[38] A. Atiser, S. Mráz, and J. M. Schneider, J. Phys. D: Appl. Phys. 42 (2009) 015202.

[39] K. Sarakinos, D. Music, F. Nahif, K. Jiang, A. Braun, C. Zilkens, and J. M. Schneider, Phys. Status Solidi RRL 4 (2010) 154.

[40] D. Music, F. Nahif, K. Sarakinos, N. Friedrichsen, and J. M. Schneider, Appl. Phys. Lett. 98 (2011) 111908.

[41] J. Robertson, Mat. Sci. Eng. R37 (2002) 129.

[42] E. Byon, T.W.H. Oates, and A. Anders, 82 (2003) 1634.

[43] V. Puri and R. K. Puri, Jpn. J. Appl. Phys.32 (1993) 4699.

[44] R. B. Patil, R. K. Puri, and V. Puri, J. All. Comp.463 (2008) 453.

[45] S. B. Lee, Phys. Rev. E. 83 (2011) 041605.

[46] G.A. Almyras, G.M. Matenoglou, Ph. Komnimou, C. Kosmidis, P. Patsalas and G.A. Evangelakis , J. Appl. Phys. 107 (2010) 084313.

[47] M. Schmid, C. Lenauer, A. Buchsbaum, F. Wimmer, G. Rauchbauer, P. Scheiber, G. Betz, and P. Varga, Phys. Rev. Lett. 103 (2009) 076101. 
[48] D.H.A. Blank, G. Koster, G. Rijnders, E. Van Setter, P. Slyke, and H. Rogalla, Appl. Phys. A: Mat. Sci. Proc. 69 (1999) S17.

[49] V. Kouznetsov, K. Macak, J.M. Schneider, U. Helmersson and I. Petrov, Surf. Coat. Technol. 122 (1999) 290

[50] D.V. Mozgrin, I.K. Fetisov, G.V. Khodachenko, Plasma Phys. Rep. 21 (1995) 401.

[51] .P. Bugaev, N.N. Koval, N.S. Sochugov, A.N. Zakharov, XVIIth International on Discharges and Electrical Insulation in Vacuum, 1996, p. 1074.

[52] I.K. Fetisov, A.A. Filippov, G.V. Khodachenko, D.V. Mozgrin, A.A. Pisarev, Vacuum 53 (1999) 133.

[53] U. Helmersson, M. Lattemann, J. Bohlmark, A.P. Ehiasarian, and J.T. Gudmundsson, Thin Solid Films 513 (2006) 1.

[54] J. Alami, S. Bolz, and K. Sarakinos, J. All. Comp. 483 (2009) 530.

[55] K. Sarakinos, J. Alami, and S. Konstantinidis, Surf. Coat. Technol. 204 (2010) 1661.

[56] A. Anders, Surf. Coat. Technol. 205 (2011) S1.

[57] D. Lundin and K. Sarakinos, J. Mater. Res. 27 (2012) 780.

[58] J.T. Gudmundsson, N. Brenning, D. Lundin, and U. Helmersson, J. Vac. Sci. Technol. A 30 (2012) 030801.

[59] K. Sarakinos, D. Music, S. Mraz, M. to Baben, K. Jiang, F. Nahif, A. Braun, C. Zilkens, S. Konstantinidis, F. Renaux, D. Cossement, F. Munnik, and J. M. Schneider, J. Appl. Phys. 108 (2010) 014904.

[60] F. Magnus, A.S. Inganson, S. Olafsson, and J. T. Gudmundsson, IEEE ELETRON DEVICE LETTERS, 33 (2012) 1045. 
[61] M. Lattemann, U. Helmersson, and J. E. Greene, Thin Solid Films 518 (2010) 5978.

[62] G. Greczynski, , J. Jensen, J. Böhlmark, and L. Hultman, Surf. Coat. Technol. 205 (2010) 118.

[63] A. P. Ehiasarian, A. Vetushka, Y. A. Gonzalvo, G. Sfrn, L. Székely, P. B. Barna, J. Appl. Phys., 109 (2011) 104314.

[64] S. Shayestehaminzadeh, R.L. Magnusson, H.P. Gislason, S. Olafsson, Thin Solid Films, 549 (2013) 172.

[65] K. Macák, V. Kouznetsov, J. Schneider, U. Helmersson and I. Petrov, J. Vac. Sci. Technol. A 18 (2000) 1533.

[66] F. Mitschker, M. Prenzel, J. Benedikt, C. Maszl, and A. von Keudell, J. Phys. D: Appl. Phys. 46 (2013) 155204.

[67] R. Koch, Surf. Coat. Technol. 204 (2010) 1973.

[68] D. Sander, J. Phys.: Condens. Matter. 16 (2004) R603.

[69] G. C. A. M. Janssen, Thin Solid Films 515 (2007) 6654.

[70] A. Fillon, G. Abadias, A. Michel, C. Jaouen, and P. Villechaise, Phys. Rev. Lett. 104 (2010) 096101.

[71] D.H. Trinh, K. Back, G. Pozina, H. Blomqvist, T. Selinder, M. Collin, I. Reineck, L. Hultman, and H. Högberg, Surf. Coat. Technol. 203 (2009) 1682.

[72] W. H. Gitzen, Alumina as a Ceramic Material, Columbus: The American Ceramic Society, 1970.

[73] F. Spaepen, Acta Materialia 48 (2000) 31.

[74] R. C. Cammarata, T. M. Trimble, and D.J. Srolovitz, J. Mater. Res. 15 (2000) 2468. 
[75] G. C. A. M. Janssen, A. J. Dammers, V. G. M. Sivel, and W. R. Wang, Appl. Phys. Lett. 83 (2003) 3287.

[76] R. W. Hoffman, Thin Solid Films 34 (1976) 185.

[77] B. W. Sheldon, A. Bhandari, A. F. Bower, S. Raghavan, X. Weng and J. M. Redwing, Acta Mater. 55 (2007) 4973.

[78] E. Chason, J. W. Shin, S. J. Hearne and L. B. Freund, J. Appl. Phys. 111 (2012) 083520.

[79] E. Chason, Thin Solid Films 526 (2012) 1.

[80] D. Flötotto, Z. M. Wang, L. P. H. Jeurgens, E. Bischoff and E. J. Mittemeijer, J. Appl. Phys. 112 (2012) 043503.

[81] A. González-González, C. Polop, and E. Vasco, Phys. Rev. Lett. 110 (2013) 056101.

[82] W.D. Nix and B.M. Clemens, J. Mater. Res. 14 (1999) 3467.

[83] S. C. Seel, C. V. Thompson, S. J. Hearne, and J. A. Floro, J. Appl. Phys. 88 (2000) 7079.

[84] L. B. Freund and E. Chason J. Appl. Phys.89 (2001) 4866.

[85] G. C. A. M. Janssen and J.-D. Kamminga, Appl. Phys. Lett. 85 (2004) 3086.

[86] J. -D. Kamminga, T. H. Keijser, R. de Delhez, and E. J. Mittemeijer, J. Appl. Phys. 88 (2000) 6332.

[87] G. Abadias, Y. Y. Tse, Ph. Guérin, and V. Pelosin, J. Appl. Phys. 99 (2006) 113519 .

[88] A. Debelle, G. Abadias, A. Michel, and C. Jaouen, Appl. Phys. Lett., 84 (2004) 5034 .

[89] A. Debelle, G. Abadias, A. Michel, C. Jaouen and V. Pelosin, J. Vac. Sci. Technol. A 25 (2007) 1438. 
[90] J. A. Floro, E. Chason, R. C. Cammarata and D. J. Srolovitz, MRS Bull. 27 (2002) 19.

[91] J. S. Tello, A. F. Bower, E. Chason and B. W. Sheldon, Phys. Rev. Lett. 98 (2007) 216104.

[92] C. Pao, S. M. Foiles, E. B. Webb, D. J. Srolovitz and J. A. Floro, Phys. Rev. B 79 (2009) 224113.

[93] R. Daniel, K. J. Martinschitz, J. Keckes and C. Mitterer, Acta Mater. 58 (2010) 2621.

[94] L. E. Koutsokeras and G. Abadias, J. Appl. Phys. 111 (2012) 093509.

[95] J. W. Shin and E. Chason Phys. Rev. Lett. 103 (2009) 056102.

[96] J. Leib, R. Mönig, and C. V. Thompson, Phys. Rev. Lett. 102 (2009) 256101.

[97] J. Leib, and C.V. Thompson. Phys. Rev. B 82 (2010) 121402.

[98] H. Z. Yu, J. Leib, S. T. Boles, and C. V. Thompson, J. Appl. Phys. 115 (2014) 043521.

[99] A. González-González, G.M. Alonzo-Medina A.I. Oliva, C. Polop, J. L. Sacedón, and E. Vasco, Phys. Rev. B 84 (2011) 155450.

[100] R. Koch, D. Hu, and A. Das, Phys. Rev. Lett. 94 (2005) 146101.

[101] C. Friesen and C. V. Thompson, Phys. Rev. Lett. 89 (2002) 126103.

[102] C. Friesen,S. C. Seel, and C. V. Thompson, J. Appl. Phys. 95 (2004) 1011.

[103] C. Friesen and C. V. Thompson, Phys. Rev. Lett. 93 (2004) 056104.

[104] C- W. Pao, D. Srolovitz, and C. V. Thompson, Phys. Rev. B 74 (2006) 155437.

[105] A. Rajamani, R. Beresford, and W. Sheldon, Appl. Phys. Lett. 79 (2001) 3776.

[106] G. Abadias and Y. Y. Tse, J. Appl. Phys. 95 (2004) 2414.

[107] JCPDS card no. 42-1120.

[108] Y. A. Kryukov and J. G. Amar, Phys. Rev. B 81 (2010) 165435.

[109] F. A. Nichols and W.W. Mullings, J. Appl. Phys. 36 (1965) 1826. 
[110] G. Jeffers, M. A Dubson and P. M. Duxbury, J. Appl. Phys. 75 (1994) 5016

[111] J. Carrey and J.-L Maurice, Phys. Rev. B 63 (2011) 245408.

[112] M. Schmid, C. Lenauer, A. Buchsbaum, F. Wimmer, G.Rauchbauer, P.

Scheiber, G. Betz, and P. Varga, Phys. Rev. Lett. 103 (2009) 076101.

[113] M. Samuelsson, D. Lundin, J. Jensen, M.A. Raadu, J.T. Gudmundsson, and U. Helmersson, Surf. Coat. Technol. 205 (2010) 591. 


\section{Figure captions}

Fig. 1. Evolution of stress-thickness product during growth of high (Ag) and lowmobility (Mo) metallic films. The incremental stress value can be calculated from the slope of the stress-thickness curve (unpublished data from Magnfält).

Fig. 2. Intrinsic stress $(\sigma)$ and the stress-free lattice parameter (a0) of Mo films grown by HiPIMS as function of peak target power density. It should be pointed out that increase of peak target power density lead to larger ion content in the deposition flux as well as to larger ion energies (data taken from Magnfält et al. [12]).

Fig. 3. Qualitative effect of temperature $(T)$ and time-averaged deposition flux $(F)$ on the island density estimated using Eqs. (1) to (3).

Fig. 4. Diffusivity regimes and scaling relations of nucleation density in pulsed deposition (after Jensen and Niemeyer [10]).

Fig. 5. Effect of pulsing frequency on the thickness at which a continuous Ag film is formed on $\mathrm{SiO}_{2}$. Data for films grown using a continuous deposition flux in the same average growth rate as that of the pulsed flux as also presented for reference (data for pulsed deposition are from Magnfält et al. [13] while data for continuous deposition are unpublished results by Elofsson) 

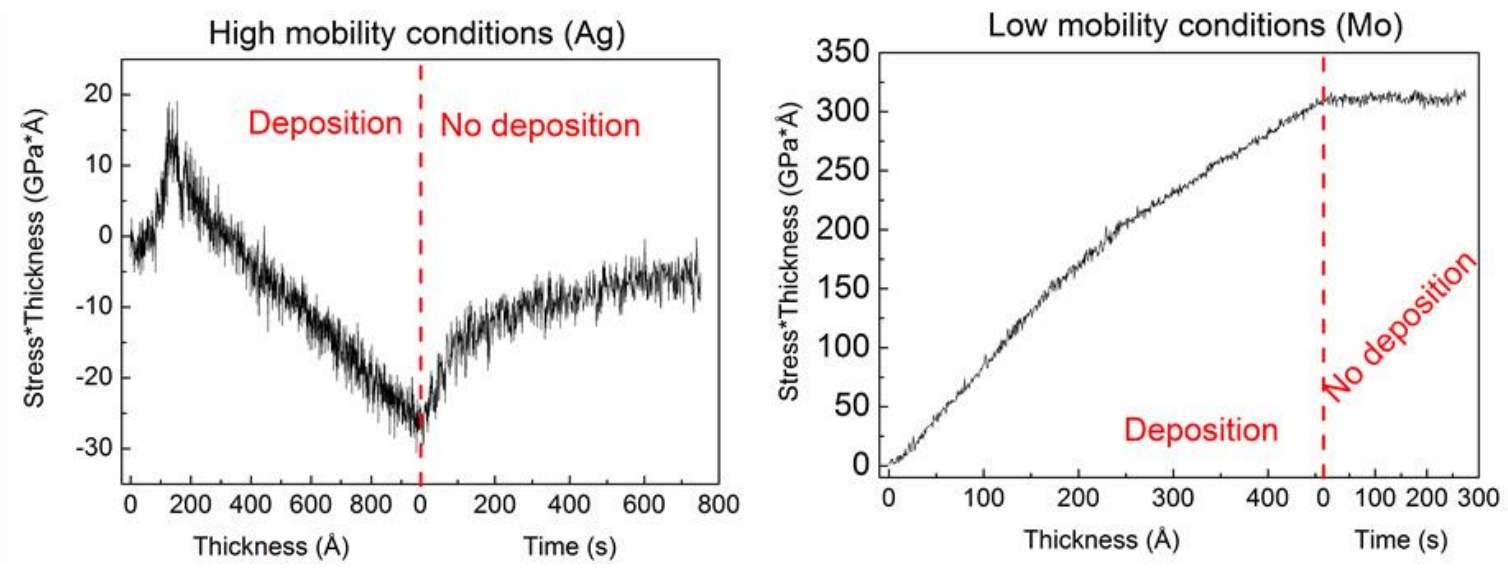

FIG.1 


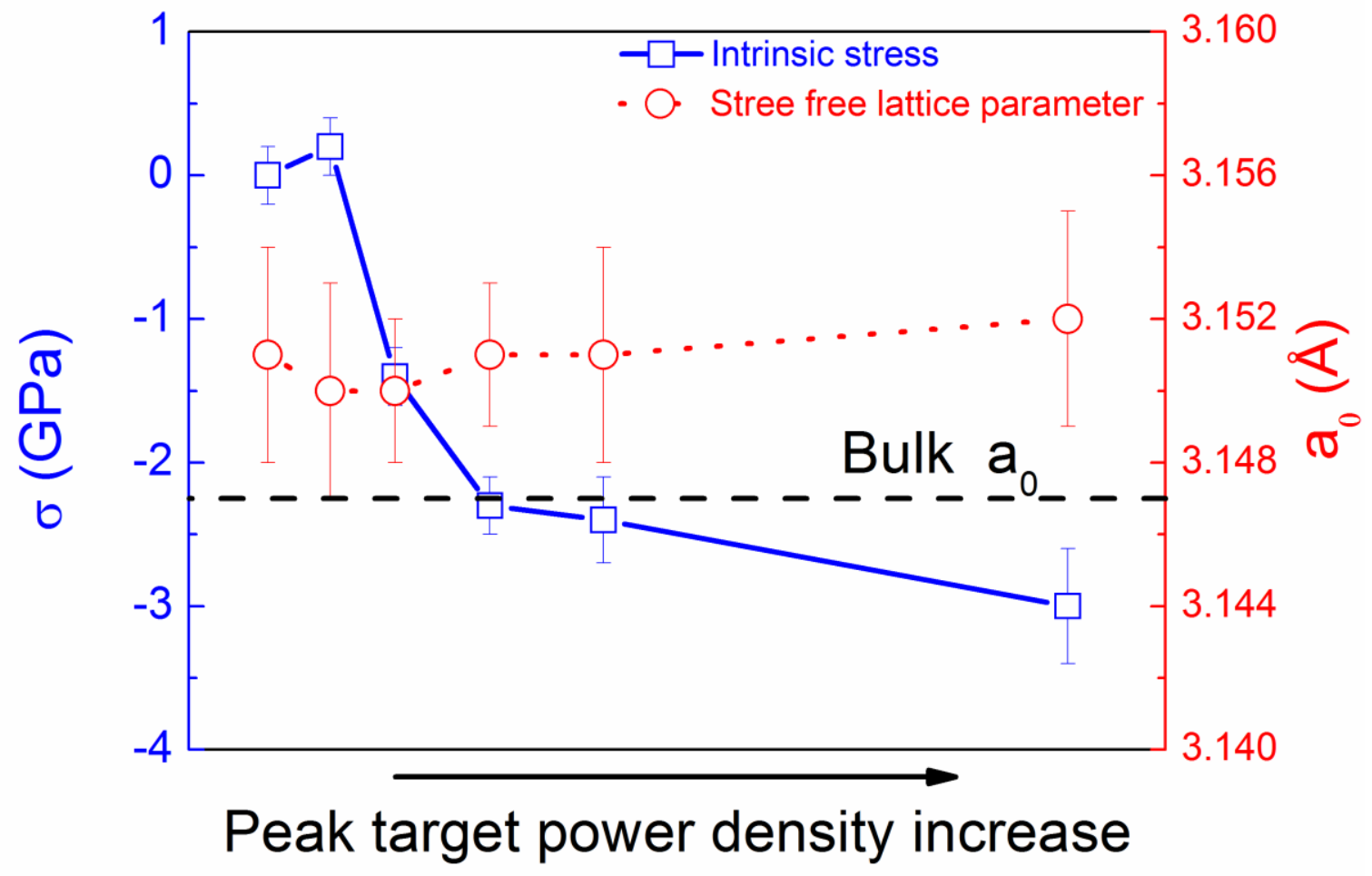

FIG.2 
$F$ [arb. units]

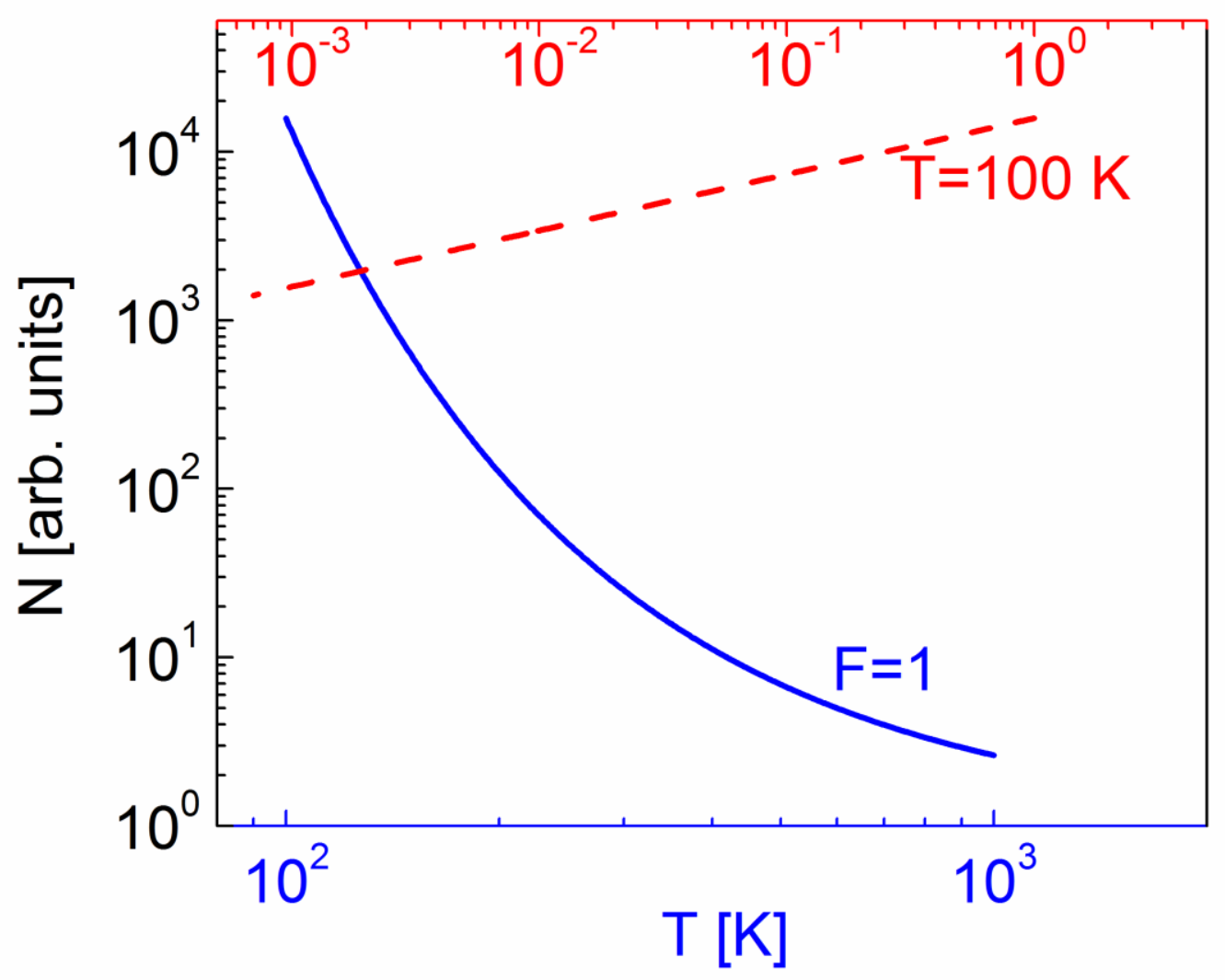

FIG.3 


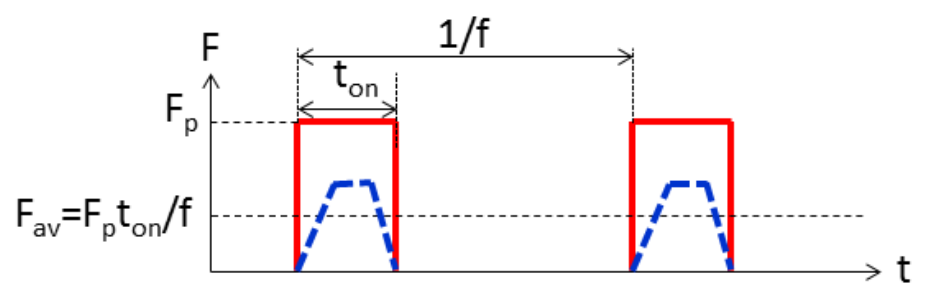

Fast diffusion $\left(\tau_{\mathrm{m}} \ll<\mathrm{t}_{\mathrm{on}}\right)$

$$
N \sim\left(F_{p} / D\right)^{1 / 3}
$$

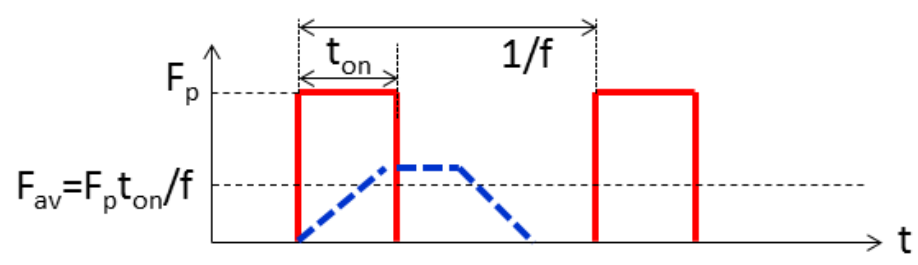

Intermediate diffusion

$$
\begin{aligned}
& \left(\mathrm{t}_{\mathrm{on}}<\tau_{\mathrm{m}}<1 / \mathrm{f}\right) \\
& \mathrm{N}^{\sim}\left(\mathrm{F}_{\mathrm{p}} \mathrm{t}_{\mathrm{on}}\right)^{1 / 2}
\end{aligned}
$$

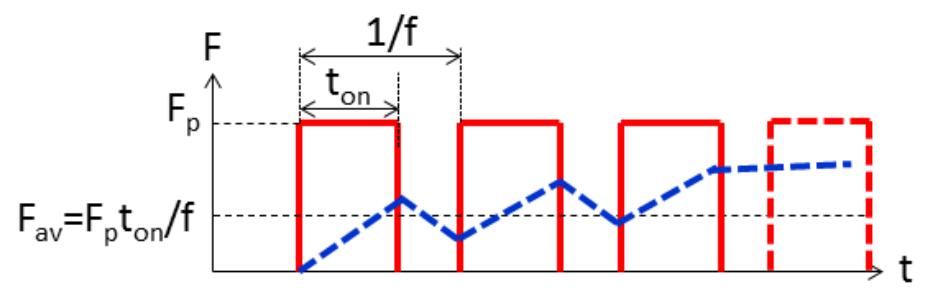

Slow diffusion $\left(\tau_{m}>>1 / f\right)$

$$
\mathrm{N} \sim\left(\mathrm{F}_{\mathrm{av}} / D\right)^{1 / 3}
$$

FIG.4

30 


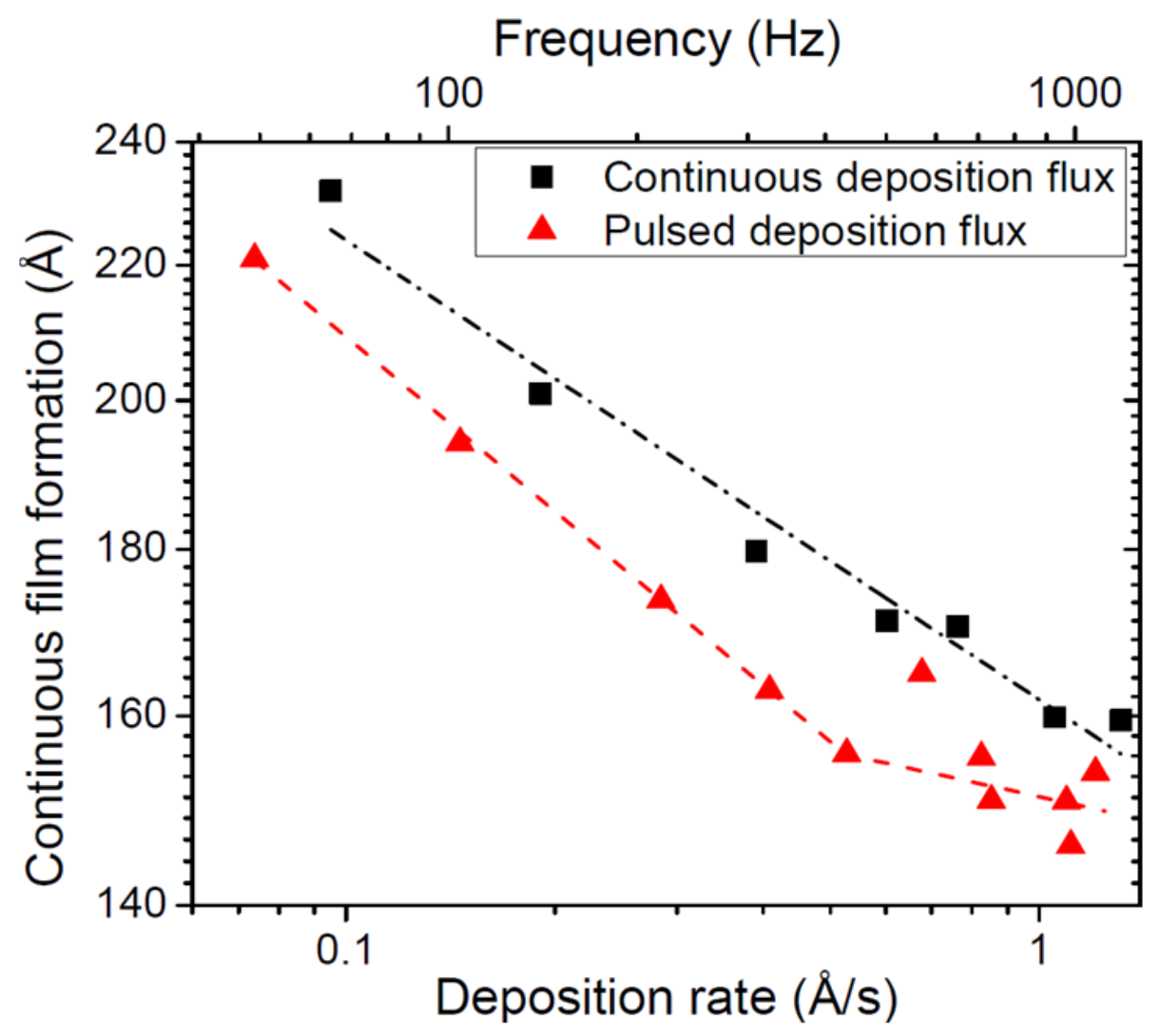

FIG.5 